\title{
Metallic islands in the Kondo insulator $\mathrm{SmB}_{6}$
}

\author{
J. C. Souza $\odot,{ }^{1,2, *}$ P. F. S. Rosa $\odot,{ }^{3}$ J. Sichelschmidt $\odot,{ }^{2}$ M. Carlone $\odot,{ }^{4}$ P. A. Venegas,${ }^{5}$ M. O. Malcolms $\odot,{ }^{1}$ P. M. Menegasso, ${ }^{1}$ \\ R. R. Urbano, ${ }^{1}$ Z. Fisk ${ }^{\circ},{ }^{6}$ and P. G. Pagliuso ${ }^{1}{ }^{1}$ \\ ${ }^{1}$ Instituto de Física “Gleb Wataghin,” UNICAMP, 13083-859, Campinas, São Paulo, Brazil \\ ${ }^{2}$ Max Planck Institute for Chemical Physics of Solids, D-01187 Dresden, Germany \\ ${ }^{3}$ Los Alamos National Laboratory, Los Alamos, New Mexico 87545, USA \\ ${ }^{4}$ POSMAT, Faculdade de Ciências, UNESP, C.P. 473, 17033-360, Bauru, São Paulo, Brazil \\ ${ }^{5}$ Departamento de Física, Faculdade de Ciências, UNESP, C.P. 473, 17033-360, Bauru, São Paulo, Brazil \\ ${ }^{6}$ Department of Physics and Astronomy, University of California, Irvine, California 92697,USA
}

(Received 2 June 2020; accepted 7 October 2020; published 3 November 2020)

\begin{abstract}
The predicted interplay between Kondo physics and nontrivial topology in $\mathrm{SmB}_{6}$ has stimulated many experimental reports, some of which are in apparent contradiction. The origin of the dispute may lie in the fragility of the Kondo insulating phase in the presence of Sm vacancies (Kondo holes) and/or natural impurities, such as $\mathrm{Gd}^{3+}$. In this work, we locally investigate this fragility for Al flux grown $\mathrm{Sm}_{1-x} \mathrm{Gd}_{x} \mathrm{~B}_{6}$ single crystals $(0 \leqslant x \leqslant 0.02)$ by combining electron spin resonance (ESR) and complementary bulk measurements. The $\mathrm{Gd}^{3+}$ ESR spectra in a highly dilute regime $(x \sim 0.0004)$ display the features of an insulating cubic environment. Remarkably, a metallic ESR line shape is observed for more concentrated samples $(x \geqslant 0.004)$, even though these systems are still in a reasonably dilute regime and show insulating dc electrical resistivity. Our data indicate that the Kondo insulating state is destroyed locally around impurities before a global percolation occurs. This result not only explains the discrepancy between $\mathrm{dc}$ and ac conductivity but also provides a scenario to explain the presence of quantum oscillations in magnetization in the absence of quantum oscillations in electrical resistivity.
\end{abstract}

DOI: 10.1103/PhysRevResearch.2.043181

\section{INTRODUCTION}

The Kondo insulator $\mathrm{SmB}_{6}$ has attracted a lot of interest for almost half a century due to numerous puzzling properties such as the physics of the hybridization gap, the mixed-valence ground state, nonzero specific heat at low temperatures, the crystalline electrical field ground state, and the saturation in the resistivity under $T \approx 4 \mathrm{~K}$ [1]. The interest was renewed following the prediction that $\mathrm{SmB}_{6}$ is a topological Kondo insulator (TKI) [2,3]. Many experimental results support a TKI phase [4-10], but this classification remains a matter of contention [11]. Conflicting experimental results for $\mathrm{SmB}_{6}$ further complicate classification schemes.

For instance, quantum oscillations in magnetization are observed in floating-zone grown samples, whereas they are absent in aluminum flux grown ones [12-14]. Electrical conductivity measurements also appear to be conflicting. A bulk-activated insulating behavior is obtained in dc electrical resistance measurements [15], while ac conductivity measurements show localized states with conductivities orders of magnitude higher than the dc measurements [16-18].

\footnotetext{
*jcsouza@ifi.unicamp.br

Published by the American Physical Society under the terms of the Creative Commons Attribution 4.0 International license. Further distribution of this work must maintain attribution to the author(s) and the published article's title, journal citation, and DOI.
}

These discrepancies naturally invite a discussion regarding the role of impurities and defects in $\mathrm{SmB}_{6}$. Raman spectroscopy measurements explored the effect of Sm vacancies and argued for a breakdown of the Kondo insulating phase for small numbers of Sm vacancies [19,20]. However, Corbino disk dc resistance measurements have shown that Sm vacancies do not affect the thermally activated bulk behavior of flux-grown $\mathrm{SmB}_{6}$, indicating that the bulk may be immune to disorder in the dc limit [15]. The effect of Sm vacancies acting as "Kondo holes" [21], i.e., an isolated nonmagnetic impurity in a Kondo lattice [22-25], also has consequences for the formation of a possible TKI phase [19]. On the one hand, low-energy spin excitons could destroy the protection of the gapless surface states [26]. On the other hand, Kondo holes are argued to enable quasiparticle interference patterns that reveal the heavy surface states in recent scanning tunneling spectroscopy (STS) measurements [27]. Furthermore, STS results showed that $\mathrm{Gd}^{3+}$ impurities in $\mathrm{SmB}_{6}$ destroy the surface states locally, and the effect percolates for $x \geqslant 0.03$ [28].

An outstanding question is whether magnetic impurities such as $\mathrm{Gd}^{3+}$, which is a natural impurity in Sm, could also display effects similar to the Sm vacancies on the hybridization gap of $\mathrm{SmB}_{6}$. In order to properly address this question, the use of a microscopic technique that locally probes the effects of $\mathrm{Gd}^{3+}$ in $\mathrm{SmB}_{6}$ is highly desirable. Recent experimental results by Fuhrman et al. were explained assuming the possibility of a dynamic screening of localized $\mathrm{Gd}^{3+}$ moments, which is unexpected due to the $\mathrm{Gd}^{3+} 4 f^{7}$ electronic 
configuration, which carries no orbital moment and is particularly stable $[29,30]$.

The $\mathrm{Gd}^{3+}$ spin is a standard probe in electron spin resonance (ESR) experiments; however, to the best of our knowledge, there is no report of ESR in highly dilute samples $(x \leqslant 0.0004)$, except the observation by Wiese et al. [31] of an anomalous spectrum of $\mathrm{Gd}^{2+}$ for concentrations down to 200 parts per million. This spectrum most likely originates from the resonance of a $\mathrm{Gd}^{2+}$ ion within an oxide impurity phase on the crystal surface [32]. Other reports are not reproducible [33,34], which begs for a revisited experimental investigation.

Here we present ESR and complementary macroscopic measurements of high-quality flux-grown $\mathrm{Sm}_{1-x} \mathrm{Gd}_{x} \mathrm{~B}_{6}$ single crystals with nominal concentrations $x=0,0.0004,0.004$, and 0.02 . From magnetic susceptibility data and using the ESR-determined actual $\mathrm{Gd}^{3+}$ concentrations, we extracted a $\mathrm{Gd}^{3+}$ magnetic moment that is close to the theoretically expected value. In the highly dilute regime $(x=0.0004)$ at $T=$ $4 \mathrm{~K}$, the $\mathrm{Gd}^{3+}$ ESR shows seven symmetrical Lorentzian lines typical of a cubic insulating environment. Increasing the $\mathrm{Gd}^{3+}$ concentration to $x=0.004$, a single asymmetrical line shape appears, which is characteristic of metallic samples for which the microwave skin depth is smaller than the sample size [35-37]. These results suggest that $\mathrm{Gd}^{3+}$ ions could close the hybridization gap locally, while the resultant metallic islands do not affect the global hybridization gap in this concentration range.

\section{METHODS}

Single-crystalline samples of $\mathrm{Sm}_{1-x} \mathrm{Gd}_{x} \mathrm{~B}_{6}$ were synthesized by the $\mathrm{Al}$ flux grown technique as described elsewhere [15]. The samples had a typical size of $\sim 700 \mu \mathrm{m}$ wide, $300 \mu \mathrm{m}$ high, and $120 \mu \mathrm{m}$ thick. Magnetic susceptibility measurements were carried out in superconducting quantum interference device (SQUID) and SQUID vibrating-sample magnetometers. Specific heat measurements were performed in a commercial small-mass calorimeter system. Electrical resistivity was measured using a standard four-point technique with a dc bridge. ESR measurements were performed on single crystals in an $X$-band $(v \cong 9.4 \mathrm{GHz})$ spectrometer equipped with a goniometer and a He flow cryostat in the temperature range of $2.6 \leqslant T \leqslant 40 \mathrm{~K}$ at very low power of $P$ $=0.21 \mathrm{~mW}$. In order to calibrate the ESR intensity in our spectrometer to estimate the actual $\mathrm{Gd}^{3+}$ concentrations, we used a standard weak-pitch sample with $0.79 \times 10^{14}$ spins $/ \mathrm{cm}^{3}$ and a $g$ value $g_{\text {ref }}=2.002(1)$. The samples were etched before the ESR measurements in a mixture of hydrochloric and nitric acids in a proportion of 3:1 to remove any possible impurities on the surface of the crystals due to $\mathrm{Al}$ flux. We did not polish any crystals in this study. The mass of the studied samples ranged from 0.3 to $4 \mathrm{mg}$. We performed our experiments on 20 different crystals from five different batches and found no sample dependence. The concentration $x$ used below refers to the nominal concentration value.

\section{RESULTS AND DISCUSSION}

Figure 1 summarizes the bulk macroscopic properties of $\mathrm{Sm}_{1-x} \mathrm{Gd}_{x} \mathrm{~B}_{6}$ with $x=0.0004,0.004$, and 0.02 . The top panel

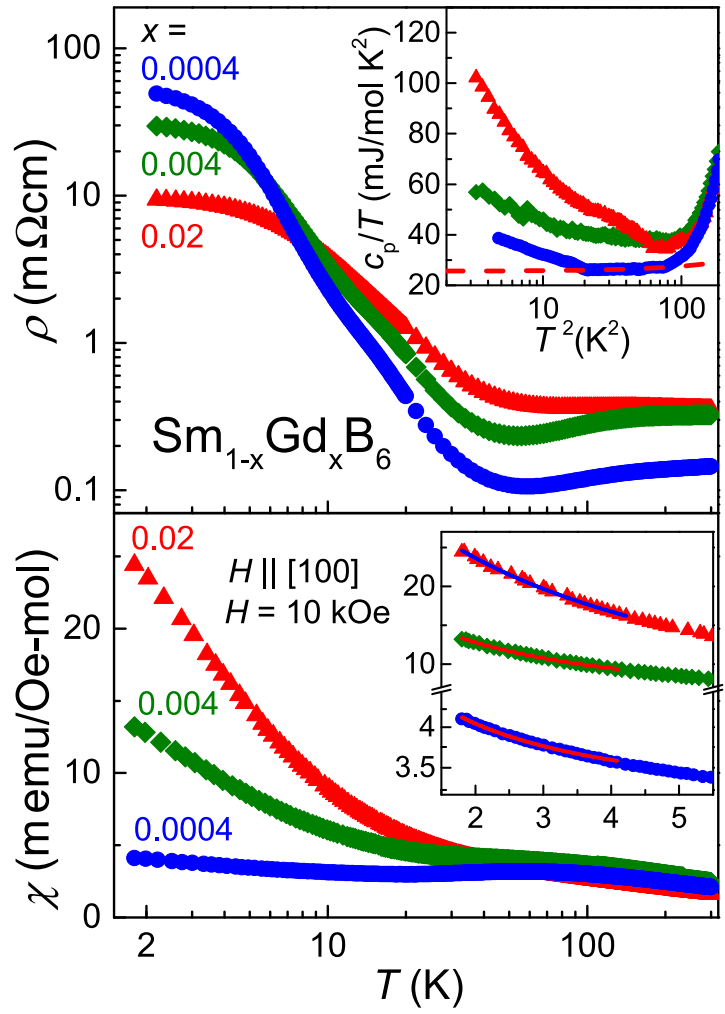

FIG. 1. Temperature dependences of dc electrical resistivity $\rho$, specific heat $c_{p}$, and dc magnetic susceptibility $\chi$ for $\operatorname{Sm}_{1-x} \mathrm{Gd}_{x} \mathrm{~B}_{6}$ with $x$ as indicated. For $\chi$, the magnetic field $H=10 \mathrm{kOe}$ is applied parallel to the [100] direction. The lower inset shows $\chi$ at low temperatures. For $\chi$, the solid lines are the best fits obtained considering a Curie-Weiss plus a Pauli $T$-independent term. For $c_{p}$ the red dashed line shows, for $x=0.0004$, how we estimated the $\gamma$ value.

of Fig. 1 shows the resistivity as a function of temperature. At low temperatures, the three concentrations show a saturation in the resistivity, consistent with the undoped compound $[5,6]$. This is expected because the saturation disappears only for $\mathrm{Gd}^{3+}$ concentrations of $x \geqslant 0.03[5,28]$. Using the value of the resistivity at $4 \mathrm{~K}$, we estimate the skin depth in a first approximation of the investigated samples to be $\delta_{D}=\sqrt{\rho / \pi f \mu} \approx 90$, 80 , and $50 \mu \mathrm{m}$ for $x=0.0004,0.004$, and 0.02 , respectively. These estimated values should be taken with extreme care as recent magnetotransport measurements have shown that subsurface cracks create additional conduction channels in $\mathrm{SmB}_{6}$, which affects the maximum resistivity value obtained [38]. A more dedicated investigation would be appropriate to compare resistivity values of our results with undoped samples. Consequently, we are probably underestimating the skin depth, which may be even larger than the sample size $(\approx 350 \mu \mathrm{m})$. Finally, it is important to notice that all samples show a similar gap in the resistivity, which agrees with previous Corbino disk measurements and the effects of $\mathrm{Sm}$ vacancies on the resistivity of $\mathrm{SmB}_{6}$ [15].

The low-temperature specific heat $c_{p}$ is shown as a function of $T^{2}$ in the inset in the top panel of Fig. 1. By extrapolating the high- $T c_{p}$ data towards $T \rightarrow 0 \mathrm{~K}$, we estimate the Sommerfeld coefficients $\gamma \sim 20,30$, and $35 \mathrm{~mJ} / \mathrm{mol} \mathrm{K}^{2}$ for $x=0.0004,0.004$, and 0.02 , respectively. The red dashed 
line exemplifies the extrapolation for $x=0.0004$. These values should be taken with care, especially comparing samples with different $\mathrm{Gd}^{3+}$ concentrations. It has been reported that undoped samples can show very distinct $\gamma$ values [14] and disorder could play a role in the Sommerfeld coefficient [39]. More importantly, the low-temperature increase of $c_{p}$ is related to a Kondo-impurity-like behavior, which is similar to previous reports for $\mathrm{Sm}_{1-x} \mathrm{La}_{x} \mathrm{~B}_{6}, \mathrm{Sm}_{1-x} \mathrm{~B}_{6}$, and $\mathrm{Sm}_{1-x} \mathrm{Gd}_{x} \mathrm{~B}_{6}$ [29,40]. Although this increase has been attributed to a local screening of the $\mathrm{Gd}^{3+}$ ions [29], the local destruction of the Kondo lattice by a nonscreened ion, which is a Kondo hole effect, also provides a reasonable explanation for this increase [41]. We should note that it appears to be additional entropy into the system when we compare $\mathrm{Sm}_{1-x} \mathrm{La}_{x} \mathrm{~B}_{6}$ and $\mathrm{Sm}_{1-x} \mathrm{Gd}_{x} \mathrm{~B}_{6}$, which was reported before [29,40]. This additional entropy, for example, might arise from antiferromagnetic (AFM) exchange field fluctuations and/or a possible $\mathrm{Gd}^{3+}$ interaction with local conduction electrons at ultralow $T$ (approximately millikelvins).

The magnetic susceptibility as a function of temperature is shown in the bottom panel of Fig. 1. A clear $x$-dependent Curie-Weiss-like behavior is observed at low temperatures, whereas at higher temperatures the behavior of the magnetic susceptibility data is consistent with undoped $\mathrm{SmB}_{6}[42,43]$. Above $T \approx 5 \mathrm{~K}$ the data start to deviate from the Curielike behavior, which means that the $\mathrm{Gd}^{3+}$ contribution is not the most dominant one. We have used the low-temperature region, $T \leqslant 4 \mathrm{~K}$, to isolate the contribution from the $\mathrm{Gd}^{3+}$ ions themselves, as shown in the inset in the bottom panel of Fig. 1.

In order to extract the magnetic moment of $\mathrm{Gd}^{3+}$ and compare it to the expected values, we have used the measured concentration $x_{\text {meas }}$ of our samples using ESR. In an ESR experiment we can estimate the number of resonant spins $N_{\text {spins }}$, comparing the ESR intensity $I$ with a well-known reference. The relationship between the sample $A$ ESR intensity and the $B$ reference can be written as [44]

$$
\frac{I_{A}}{I_{B}}=\frac{N_{\text {spins }}^{A}}{N_{\text {spins }}^{B}}\left(\frac{g_{A}}{g_{B}}\right)^{2}\left[\frac{S_{A}\left(S_{A}+1\right)}{S_{B}\left(S_{B}+1\right)}\right]\left(\frac{T_{B}}{T_{A}}\right),
$$

where $g$ is the $g$ value, $S$ is the spin moment, and $T$ is the temperature. The ESR intensity $I$ is obtained by double integrating our $\mathrm{Gd}^{3+}$ ESR spectrum. The $g$ value is a parameter extracted from the $\mathrm{Gd}^{3+}$ ESR resonance field, which is obtained by fitting our $\mathrm{Gd}^{3+}$ ESR spectrum using an admixture of absorption and dispersive derivatives. We will discuss the $\mathrm{Gd}^{3+}$ ESR $g$ value in more detail below. Figure 2 shows the $\mathrm{Gd}^{3+}$ ESR spectra for $\mathrm{Sm}_{1-x} \mathrm{Gd}_{x} \mathrm{~B}_{6}$ with $x=0$ and 0.0004 at $4 \mathrm{~K}$. The $\mathrm{Gd}^{3+} \mathrm{ESR}$ spectrum for $x=0.0004$ is resolved; that is, it contains seven distinct Lorentzian line shapes. This spectrum is the resonance of the $\mathrm{Gd}^{3+}$ fine structure $(S=7 / 2$, selection rule $\Delta m= \pm 1$ ) and is characteristic of an insulating environment having a skin depth larger than the sample size. The red solid line is a simulation considering a cubic crystal field spin Hamiltonian with a crystal field parameter (CFP) $b_{4}=-9.5(3)$ Oe [37]. Even though the $\gamma$ values obtained are not the smallest reported in the literature for $\mathrm{SmB}_{6}$ samples, they do not affect our results. The insulating $\mathrm{Gd}^{3+} \mathrm{ESR}$ line shape reinforces the clear insulating bulk environment

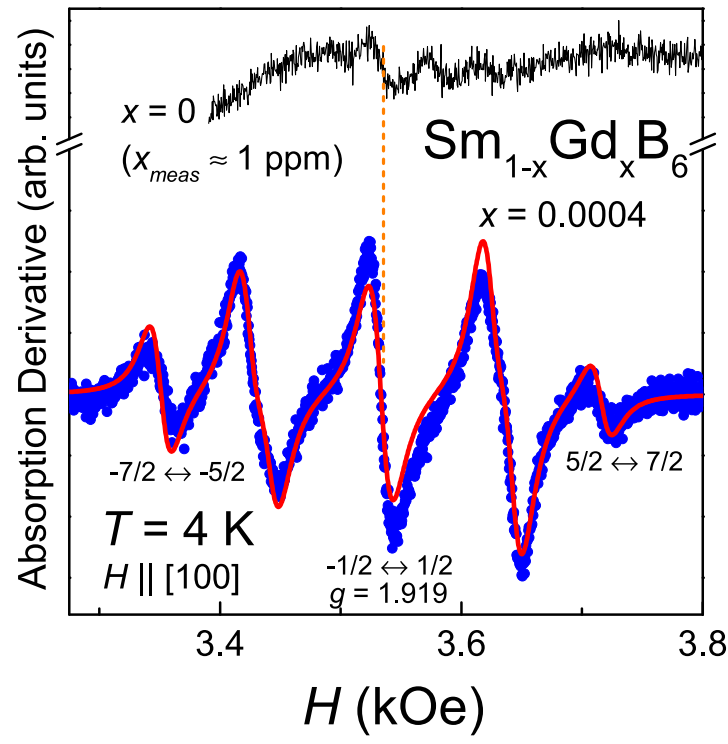

FIG. 2. $X$-band $\mathrm{Gd}^{3+}$ ESR spectra for $\mathrm{Sm}_{1-x} \mathrm{Gd}_{x} \mathrm{~B}_{6}$ single crystals with $x=0$ and 0.0004 . The field $H$ is applied parallel to the [100] direction. The dashed line shows the $g$ value of the $-1 / 2 \leftrightarrow 1 / 2$ resonance. The red solid line is a simulation assuming a cubic insulator spin Hamiltonian with a CFP $b_{4}=-9.5(3)$ Oe [37].

in our samples. For the undoped compound we were able to observe a resonance with a very similar $g$ value compared with $x=0.0004$, as shown by the orange dotted line in Fig. 2 . This is an indication that this resonance could, most likely, be coming from $\mathrm{Gd}^{3+}$ or $\mathrm{Eu}^{2+}$ natural impurities in the undoped compound. Using Eq. (1), the measured concentration $x_{\text {meas }}$ estimated was $\approx 1$ part per million, which is extremely low and shows the good quality of the undoped single crystals. We also did not observe an ESR signal from disordered $\mathrm{Sm}^{3+}$ as reported by previous studies $[45,46]$.

For the $\mathrm{Gd}^{3+}$-doped compound ESR spectra, as shown in Fig. 2, using Eq. (1), we were able to estimate the actual concentrations of $\mathrm{Gd}^{3+}$ based on $N_{\text {spins }}: x_{\text {meas }}=0.00034(1)$, $0.0039(1)$, and $0.0189(1)$. Using these values, we fit the $\chi$ data for different concentrations with a Curie-Weiss law plus a Pauli $T$-independent term in the range of $2 \leqslant T \leqslant 4 \mathrm{~K}$ (see the inset in the bottom panel of Fig. 1). The obtained magnetic moments are $\mu_{\mathrm{Gd}}=8.0(1) \mu_{B} / \mathrm{Gd}, 8.2(1) \mu_{B} / \mathrm{Gd}$, and 7.94(2) $\mu_{B} / \mathrm{Gd}$ for $x=0.0004,0.004$, and 0.02, respectively. Thus, the full theoretically expected moments for $\mathrm{Gd}^{3+}$ are observed.

Our $\mathrm{Gd}^{3+}$ ESR spectra as displayed in Fig. 2 do not support the scenario of a $\mathrm{Gd}^{3+}$ dynamic screening. The ESR should be observable only for temperatures above the single-impurity Kondo regime $\left(T \gg T_{K}\right)$, for which the $\mathrm{Gd}^{3+}$ local moment is well defined [47]. Furthermore, we do not observe the ESR spin dynamics expected for a single-impurity Kondo ion [47] or for a Kondo ion lattice [48,49]. Therefore, these results do not corroborate the scenario of dynamic $\mathrm{Gd}^{3+}$ screening proposed by Fuhrman et al. [29].

Besides supplying information on spin dynamics, the $\mathrm{Gd}^{3+}$ ESR linewidth $\Delta H$ also provides information on disorder and sample quality. For $x=0.0004$, the $\mathrm{Gd}^{3+}$ resonance of the central line at $T=4 \mathrm{~K}$, which corresponds to the $-1 / 2 \leftrightarrow$ $1 / 2$ transition, has a linewidth $\Delta H=17$ (2) Oe. When the fine 


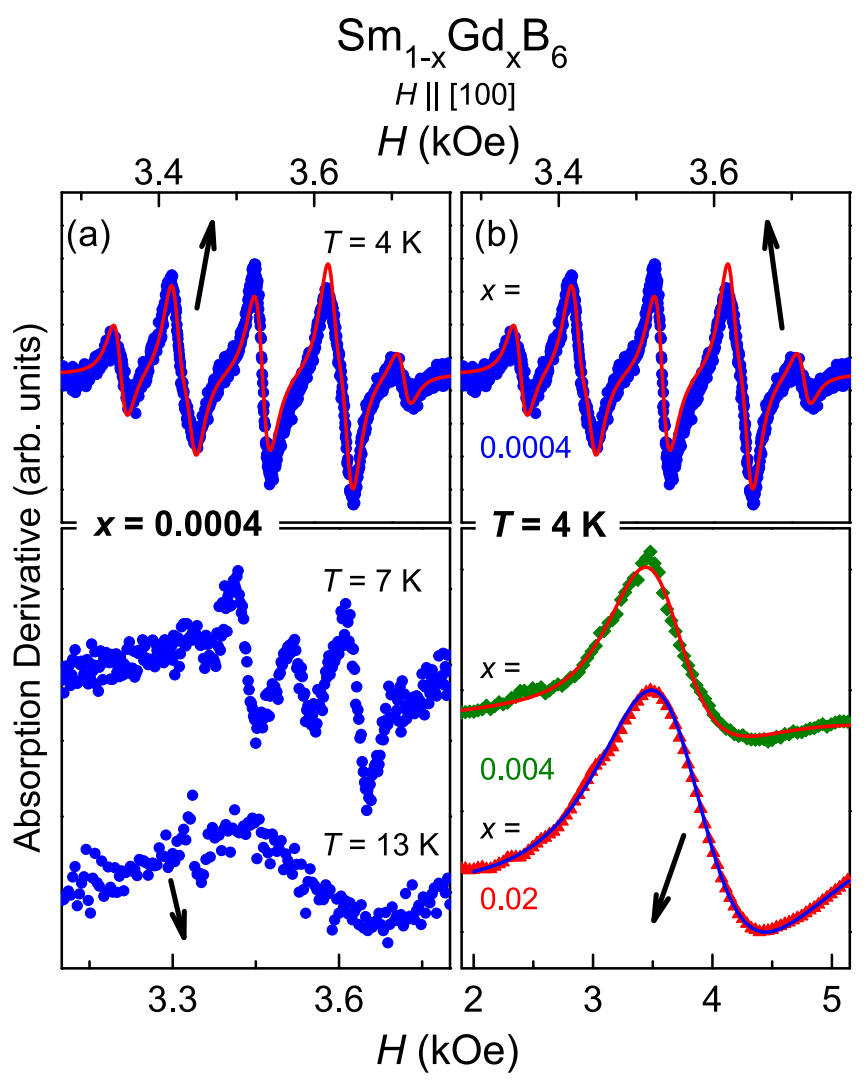

FIG. 3. $\mathrm{Gd}^{3+} X$-band ESR spectra (a) $T$ and (b) $x$ dependences for $\mathrm{Sm}_{1-x} \mathrm{Gd}_{x} \mathrm{~B}_{6}$, with $x$ indicated in the panels. The field $H$ is applied parallel to the [100] direction. The solid lines are the best fit obtained to the spectra using an admixture of absorption and dispersion derivatives for $x=0.004$ and 0.02 [37]. For $x=0.0004$ the same fit as in Fig. 2 is shown.

structure is collapsed into one resonance by varying the angle with respect to the applied magnetic field $H$ [37,44], we obtain $\Delta H=19$ (2) Oe [Fig. S2(a)]. This is consistent with samples known as good insulators [50]. Further, the $\mathrm{Gd}^{3+}$ linewidth at $T=4 \mathrm{~K}$ has a similar value when compared with previous results for $\mathrm{Er}^{3+}$-doped $\mathrm{SmB}_{6}[42,51]$.

Figure 3(a) shows the $\mathrm{Gd}^{3+}$ ESR line shape evolution as a function of temperature for $\mathrm{Sm}_{1-x} \mathrm{Gd}_{x} \mathrm{~B}_{6}$ for $x=0.0004$. As temperature increases, the seven lines merge into one line, which is a narrowing due to the interaction of the $\mathrm{Gd}^{3+} 4 f$ local moments and the conduction electrons, known as exchange narrowing [37,44,52]. However, as has already been shown for Kondo insulators [53,54], it is necessary to take into account the influence of the valence fluctuation of $\mathrm{Sm}^{2.6+}$ at the $\mathrm{Gd}^{3+}$ site to fully describe the narrowing effect.

The most intriguing change in the line shape occurs when, at constant temperature, the $\mathrm{Gd}^{3+}$ concentration is increased from $x=0.0004$ to 0.004 and 0.02. As shown in Fig. 3(b), the $\mathrm{Gd}^{3+}$ ESR spectra at $T=4 \mathrm{~K}$ for $x=0.004$ and 0.02 display a single asymmetrical Lorentzian line shape, also known as Dysonian line shape [35-37], with no $\mathrm{Gd}^{3+}$ fine structure. This line shape is characteristic of a metallic environment, where the skin depth is much smaller than the thickness of the sample. At the same temperature, the $x=0.0004$ sample still displays the seven distinct Lorentzian line shapes expected for an insulator. Therefore, the local environment surrounding the $\mathrm{Gd}^{3+}$ ions is changing as a function of the $\mathrm{Gd}^{3+}$ concentration. At the same time this is not indicated in resistivity data, where the thermally activated bulk behavior still remains. As shown in the top panel of Fig. 1, both the $T$ dependence of the resistivity data and skin depth are very similar for all concentrations, especially when we compare $x=0.0004$ and 0.004 , showing a similar indirect gap without any apparent change in the insulating ground state.

The remarkable change in the local $\mathrm{Gd}^{3+}$ environment happens between $x=0.0004$ and 0.004 . In order to understand the origin of such evolution, it is useful to compare it with nonmagnetic insulator $\mathrm{Gd}^{3+}$ - and $\mathrm{Eu}^{2+}$-doped $\mathrm{CaB}_{6}$ [55-57], where the Kondo effect does not play a role.

In $\mathrm{CaB}_{6}$, the local insulator-to-metal evolution as a function of $\mathrm{Gd}^{3+}$ was interpreted as the introduction of an extra electron to the system, with $\mathrm{Gd}^{3+}$ ions acting as electron donors and creating a hydrogenlike bound state within the gap. The bound states overlap and form a percolative network; that is, there is a change in the ground state of the system. In that case, for higher $\mathrm{Gd}^{3+}$ concentrations $(0.003 \leqslant x \leqslant$ $0.01)$, the ESR results were always consistent with the metallic resistivity data [55]. This is in contrast to the situation for $\mathrm{Gd}^{3+}$-doped $\mathrm{SmB}_{6}$, as stated above. The introduction of $\mathrm{Eu}^{2+}$ in $\mathrm{CaB}_{6}$ gives rise to a localized split-off bound state, with the Fermi energy lying in the gap of the semiconductor. This impurity state percolates only with $x \geqslant 0.3$, when the ground state becomes a ferromagnetic metal; however, it is possible to observe an $\mathrm{Eu}^{2+}$ Dysonian line shape for $x \geqslant 0.15[56,57]$.

$\mathrm{SmB}_{6}$ is not expected to host a hydrogenlike doping mechanism [58]. Furthermore, the nonparabolic band structure of $\mathrm{SmB}_{6}$ leads to a significant increase of the calculated density of impurities required for percolation [59]. Hence, the origin of the insulator-to-metal evolution in $\mathrm{SmB}_{6}$ should differ from that in $\mathrm{CaB}_{6}$. Our results agree with these two propositions. If the origin of the metallic islands and consequent evolution were due to a $\mathrm{Gd}^{3+}$-induced hydrogenlike in-gap bound state, we should expect an evolution from insulating to metallic resistivity, i.e., a change in the ground state of the compound, or a striking change in the hybridization gap value, which is not observed.

Remarkably, the insulator-to-metal evolution is also noticed in the ESR response of $\mathrm{Sm}_{1-y} \mathrm{Eu}_{y} \mathrm{~B}_{6}$ samples. The $\mathrm{Eu}^{2+}$ ESR spectrum of $y=0.0004$ shows a cubic insulator environment, with a well-defined $\mathrm{Eu}^{2+}$ hyperfine structure and a CFP $b_{4} \approx-50$ Oe [43]. For higher concentrations, e.g., $y=0.01$, the $\mathrm{Eu}^{2+}$ fine structure collapses, and we obtain a Dysonian line shape. Previous reports have shown that a metallic antiferromagnetic ground state for $\mathrm{Sm}_{1-y} \mathrm{Eu}_{y} \mathrm{~B}_{6}$ appears only at or above $y=0.4$, at least one order of magnitude higher than what it is observed by electron spin resonance [60]. The $\mathrm{Gd}^{3+}$ - and $\mathrm{Eu}^{2+}$-doped $\mathrm{SmB}_{6}$ results suggest that there is not a percolation in the system, but a local effect which does not affect the global hybridization gap.

One alternative scenario is that the $\mathrm{Gd}^{3+}$ ions are not screened by conduction electrons, although they occupy a Sm site. Instead of screening, these substitutions locally close the hybridization gap at the $\mathrm{Gd}^{3+}$ site, increasing the local density of states and/or the local number of carriers. As already stated, this Kondo hole effect of $\mathrm{Gd}^{3+}$ is consistent with the 
low- $T$ magnetic susceptibility and specific heat measurements shown in Fig. 1, as well with the scaling proposed by Fuhrman et al. [29]. Such an effect could have two different local manifestations: the first one, which is more unlikely due to the small amount of $\mathrm{Gd}^{3+}$, resides in the possibility of local in-gap impurity states $[17,18]$.

The natural scenario we propose is related to the formation of a bound state at the $\mathrm{Gd}^{3+}$ site. This state should affect locally the hybridization gap around the $\mathrm{Gd}^{3+}$ ions due to translational symmetry breaking. In other words, this substitution should create a spatial oscillation of the hybridization gap near the impurity [24]. In the highly diluted case, such metallic islands are small and separated, in the sense that the $\mathrm{Gd}^{3+}$ spins have a spin relaxation mechanism mediated by phonons. Hence, we obtain a $\mathrm{Gd}^{3+}$ ESR spectrum as expected for a cubic insulating environment. Based on previous theoretical reports [24], we propose that when there is quantum interference between such metallic islands, due to the increase of $\mathrm{Gd}^{3+}$ concentration, effectively, the islands grow in size and, as a consequence, the carriers will have mobility to enable metallic properties in the ESR line shape and ESR line parameters, which will lead to a local effect and will not reflect in the global properties of the system, such as dc resistivity. Naturally, a percolation should occur at higher concentrations. While for $\mathrm{Gd}^{3+}$-doped $\mathrm{SmB}_{6}$ we know that samples with $x=0.05$ still present an insulating behavior [29], $\mathrm{La}^{3+}$-doped samples will show a metallic-insulating transition at $x \sim 0.3$ [40]. $\mathrm{Eu}^{2+}$-doped samples have a metallic-insulating transition at $x \sim 0.4[60]$.

Despite the local change in the environment, a more systematic in-depth theoretical investigation might need to introduce inhomogeneous effects, which may arise due to $\mathrm{Gd}^{3+}$ ions in different magnetic states [44]. Such effects may distort the ESR line shape asymmetry and linewidth. Although inhomogeneous effects should, in principle, play a role in fully describing the $\mathrm{Gd}^{3+}$ ESR spectrum evolution, the possibility of new mechanisms should be a motivation for further theoretical works.

The intriguing question arising from the Kondo holes physics is related to the effects of metallic islands in a TKI. Would the metallic islands have a nontrivial topology? From an ESR viewpoint, the fingerprint of a nontrivial topological state could be related to the appearance of an ESR diffusivelike line shape for the signal of the ESR local moment probe [61]. However, one of the main ingredients for such an unusual effect is a phonon-bottleneck regime, which is not present in these studied $\mathrm{Gd}^{3+}$ concentrations. Therefore, we cannot extract any information about the topology of the system from an ESR perspective for the studied samples. Further experiments exploring different $\mathrm{Gd}^{3+}$ concentrations could be valuable in order to clarify this pressing question from our results.

In ESR, the linewidth $\Delta H$ is proportional to $1 / T_{2}$, where $T_{2}$ is the spin-spin relaxation time. Hence, using an admixture of absorption and dispersive derivatives to fit the $\mathrm{Gd}^{3+}$ ESR line shapes, we can extract $\Delta H$ and obtain information about the interaction between the $\mathrm{Gd}^{3+} 4 f$ local moments and their environment; that is, we can extract the ESR spin dynamics. The solid lines in Fig. 3(b) indicate the fit for $x=0.004$ and 0.02 . In order to exclude crystal field effects, the $T$ dependence

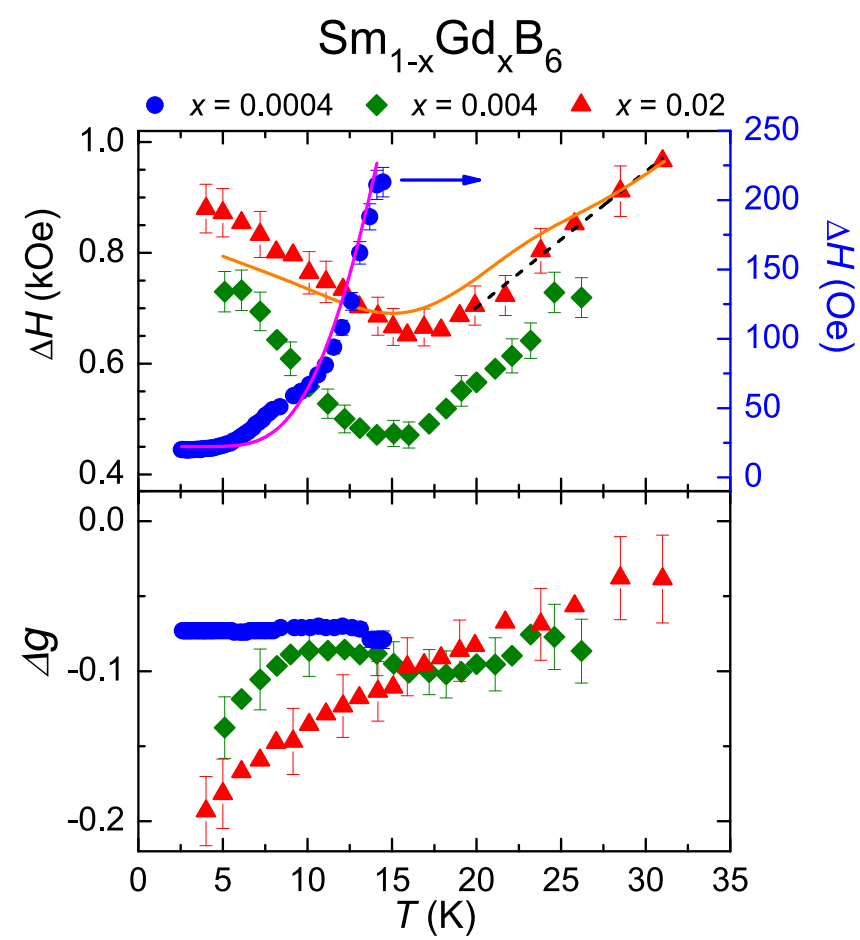

FIG. 4. Top: $T$ dependence of the $\mathrm{Gd}^{3+}$ ESR linewidth $\Delta H$ for the $\mathrm{Sm}_{1-x} \mathrm{Gd}_{x} \mathrm{~B}_{6}$ compound with $x=0.0004,0.004$, and 0.02. While the external magnetic field was applied into the [100] direction for $x=0.004$ and 0.02 , for $x=0.0004$ the magnetic field was applied $30^{\circ}$ from the [100] direction in the (110) plane (collapsed ESR spectrum). The solid lines are fits explained in the text. The dotted line is a guide to the eye. Bottom: $\Delta g$ as a function of temperature.

of $\Delta H$ for $x=0.0004$ was obtained when the $\mathrm{Gd}^{3+} \mathrm{ESR}$ spectrum is collapsed into one resonance [44]. The top panel of Fig. 4 shows the evolution of the linewidth $\Delta H$ as a function of temperature.

For the highly diluted $x=0.0004$ sample, it is possible to observe an exponential dependence of the $\mathrm{Gd}^{3+}$ ESR linewidth as a function of temperature. The interconfigurational fluctuation model (ICF) [62-64] shows that the fluctuation between the $4 f^{n}$ and $4 f^{n+1}$ configurations of the $\mathrm{Sm}$ ions provokes a fluctuating field at the $\mathrm{Gd}^{3+}$ site, which explains the exponential increase of $\Delta H$,

$$
\Delta H=\Delta H_{0}+b T+A e^{-E_{\mathrm{ex}} / T},
$$

where $\Delta H_{0}$ is the residual $\mathrm{Gd}^{3+}$ linewidth, $b$ is the Korringa relaxation rate [37,44], $E_{\mathrm{ex}}$ is the excitation energy of the $\mathrm{Sm}$ ions, and $A$ is a constant. The Korringa relaxation $b$ is related to the spin-flip scattering relaxation mechanism which arises from the interaction between conduction electrons and the $\mathrm{Gd}^{3+} 4 f$ local moments [37,44]. We obtain $b=0 \mathrm{Oe} / \mathrm{K}$ and $E_{\mathrm{ex}}=56 \mathrm{~K}$ (magenta solid line), which is, notably, of the order of the hybridization gap [15]. This result is another microscopic hint that $\mathrm{Gd}^{3+}$ ions are not introducing extrinsic charge in-gap states when substituting $\mathrm{Sm}$ ions in $\mathrm{SmB}_{6}$.

Comparing the linewidth $\Delta H$ for all samples, we can see a clear difference between the highly dilute system $(x=$ 0.0004) and the other two $\mathrm{Gd}^{3+}$ concentrations. The relaxation changes dramatically when the $\mathrm{Gd}^{3+}$ sites start to 
interact, which can be verified in trying to use the same ICF model for $x=0.02$ (orange solid line). In this case, considering a phenomenological exchange interaction between $\mathrm{Gd}^{3+}$ sites with an internal exchange field $H_{\mathrm{ex}}=20$ Oe [63], we obtain a Korringa relaxation rate $b=1.5(5) \mathrm{Oe} / \mathrm{K}$ and $E_{\text {ex }}=110(10) \mathrm{K}$, which are two times larger than those for $x=0.0004$. This clearly indicates that the model does not necessarily apply for higher $\mathrm{Gd}^{3+}$ concentrations, where the correlation between the metallic islands covers the effect of interconfigurational fluctuations.

As already mentioned, from the admixture of absorption and dispersive derivative fitting we can also extract the $\mathrm{Gd}^{3+}$ resonance field $H_{0}$. Consequently, we obtain the $\mathrm{Gd}^{3+}$ experimental $g$ value $g_{\exp }=h v / \mu_{B} H_{0}$, where $h$ is the Planck constant and $\mu_{B}$ is the Bohr magneton. The bottom panel of Figure 4 shows the temperature dependence of $\mathrm{Gd}^{3+}$, $\Delta g=g_{\exp }-g_{\text {insulator }}$, where $g_{\text {insulator }}=1.993(1)$ for an isolated $\mathrm{Gd}^{3+}$ ion [44]. In metals, for the simplest scenario [65-67], the $g$ shift $\Delta g$ is given by $\Delta g=J_{f s} \eta\left(E_{F}\right)$ [37], where $J_{f s}$ is the effective exchange interaction between the $4 f$ local moments and the conduction electrons and $\eta\left(E_{F}\right)$ is the DOS at the Fermi level for one spin direction. Positive $g$ shifts are expected due to the ferromagnetic (atomic) interaction between the $4 f$ local moments and $s$ and/or $d$ conduction electrons. Negative $g$ shifts are obtained when the contribution at the Fermi level comes from $p$ and/or $f$ conduction electrons since their magnetic interaction with the $4 f$ local moments occurs via a virtual bound state [68] and, consequently, an antiferromagnetic (covalent) exchange interaction appears $[37,68]$. In the simplest scenario for metals it is crucial to note that the Korringa relaxation $b$, which is extracted from $\Delta H T$ dependence, is also proportional to the exchange interaction between the $4 f$ local moments and the conduction electrons. In other words, $b \propto J_{f s}^{2} \eta^{2}\left(E_{F}\right)$ when we neglect $\mathbf{q}$ dependence or multiband effects [37]. Another crucial point is that internal ferromagnetic or antiferromagnetic fields can also shift the resonance field, generating apparent positive or negative $g$ shifts.

$\Delta g=-0.074(2)$ for $x=0.0004$ is $T$ independent. Since the Korringa relaxation at this concentration is negligible and the spin relaxation is phonon mediated, this negative value most likely is due to the interaction between the $\mathrm{Gd}^{3+}$ and $\mathrm{Sm}^{2.6+} 4 f$ electrons. In other words, our results indicate that there is no relevant overlap between the $\mathrm{Gd}^{3+} 4 f$ and conduction electron wave functions at these investigated temperatures, which is in agreement with our scenario. Since the magnetic contribution of the $\mathrm{Sm}^{2.6+}$ is $T$ independent at this temperature range, it behaves like a constant AFM internal field at the $\mathrm{Gd}^{3+}$ site, which is consistent with recent magnetic dichroism results [69]. In this scenario, the AFM net local field at the $\mathrm{Gd}^{3+}$ site would be $H^{\text {net }}=130(5)$ Oe.

For the higher-concentration samples there is a clear decrease of the $\mathrm{Gd}^{3+} \Delta g$ towards low temperatures. This is an indication of an exchange interaction between the $\mathrm{Gd}^{3+}$ sites, which is expected to be negative since $\mathrm{GdB}_{6}$ is an antiferromagnetic compound with $T_{N} \approx 15 \mathrm{~K}$ [70]. The possibility of $\mathrm{Gd}-\mathrm{Gd}$ interactions for such small $\mathrm{Gd}^{3+}$ concentrations $(x=0.004)$ is also suggested by STS studies which show that defects in $\mathrm{SmB}_{6}$ have a "healing length" of $\sim 2 \mathrm{~nm}$ [28]. If we assume that this would also characterize the bulk and not just the surface, the affected volume around a defect could be of the order of $\sim 500$ unit cells. It is worth noting that Gd-Gd interaction for $x=0.004$ and 0.02 is also reflected in the low- $T$ increase of the linewidth, as shown in the top panel of Fig. 4, although this increase does not scale with the $\mathrm{Gd}^{3+}$ concentration.

As discussed above, due to the correlation between the metallic regions, the local density of carriers may become mobile and start to play a role in the relaxation mechanism, resulting in a finite value of the Korringa relaxation rate even at lower temperatures for $x=0.004$ and 0.02 . As a consequence, the $\mathrm{Gd}^{3+}$ ESR turns into a single Dysonian line shape with a small Korringa relaxation; that is, the environment changes from insulatorlike to a metalliclike. Although these local effects can be seen, the global hybridization gap is not affected, as shown by dc resistivity data.

This ESR scenario helps us to understand the apparent discrepancy between ac and dc conductivity data [15-18,38]. The local response of the metallic islands will not affect the global hybridization gap due to the lack of percolation; hence, at the dc limit it is only possible to observe an insulating behavior. Recent theoretical models argue that such localized states could produce quantum oscillations in the magnetization, which is a plausible way to understand the presence of quantum oscillations in magnetization and the absence in resistivity $[12,38]$. Defects, such as Sm vacancies and natural magnetic impurities, are responsible for localized metallic islands, which allegedly could produce such oscillations [71]. Although recent theoretical results indicate that the reported oscillations could be supported by the bulk band structure of this compound [72], our results are also consistent with the emergence of quantum oscillations from bulk impurities [71]. In contrast to this theoretical proposition [72], we should note that $\mathrm{SmB}_{6}$ does not show a magnetic ordered state at ambient pressure $[73,74]$. Finally, the Kondo hole scenario for $\mathrm{Gd}^{3+}$ is also valid for $\mathrm{Eu}^{2+}$-doped $\mathrm{SmB}_{6}$ and is a plausible way to explain the scaling as a function of $\mathrm{Gd}^{3+}$ concentration in $\mathrm{SmB}_{6}$ described by Fuhrman et al. [29,41].

Our results show that there is a local insulator-to-metal evolution in $\mathrm{Gd}^{3+}$-doped $\mathrm{SmB}_{6}$ at very low concentrations. This observation was possible only because we are directly probing the $\mathrm{Gd}^{3+}$ in the bulk locally.

\section{CONCLUSION}

In summary, we performed electron spin resonance and complementary macroscopic measurements in the Kondo insulator $\mathrm{Sm}_{1-x} \mathrm{Gd}_{x} \mathrm{~B}_{6}$ with $x=0,0.0004,0.004$, and 0.02. The $\mathrm{Gd}^{3+}$ ESR spectra at $4 \mathrm{~K}$ for different concentrations showed two clearly different behaviors. For $x=0.0004$ we observed an insulatorlike $\mathrm{Gd}^{3+}$ ESR line shape, while for $x=0.004$ and 0.02 we obtained a Dysonian metallic line shape, characteristic of a conductive environment. Hence, the hybridization gap at the $\mathrm{Gd}^{3+}$ site collapses, which can result in an effective formation of larger metallic islands as a function of magnetic impurity concentration. This scenario is consistent with the observed ESR spin dynamics and shift of ESR $g$ factors. These localized states explain the discrepancy between dc and ac conductivity measurements and can also provide a different point of view regarding quantum oscillations in this 
system. Further experiments, such as ESR in "neutral"doping samples, e.g., $\mathrm{Gd}^{3+}-\mathrm{Sr}^{2+}$ and $\mathrm{Eu}^{2+}-\mathrm{La}^{3+}$ dopings, and NMR measurements in $\mathrm{Gd}^{3+}$-doped $\mathrm{SmB}_{6}$ could be valuable to understand the evolution of the metallic islands in more detail.

\section{ACKNOWLEDGMENTS}

We acknowledge constructive discussions with C. Rettori and S. M. Thomas. This work was supported by
FAPESP (São Paulo, Brazil) Grants No. 2018/11364-7, No. 2017/10581-1, No. 2016/14436-3, No. 2013/17427-7, No. 2012/04870-7, and No. 2012/05903-6; CNPq Grants No. 309483/2018-2, No. 442230/2014-1, No. 304649/2013-9, and No. 141026/2017-0; CAPES; and FINEP-Brazil. Z.F. acknowledges the funding from NSF Grant No. 1708199. Work at Los Alamos National Laboratory (LANL) was performed under the auspices of the U.S. Department of Energy, Office of Basic Energy Sciences, Division of Materials Science and Engineering.
[1] M. Dzero, J. Xia, V. Galistski, and P. Coleman, Annu. Rev. Condens. Matter Phys. 7, 249 (2016).

[2] M. Dzero, K. Sun, V. Galitski, and P. Coleman, Phys. Rev. Lett. 104, 106408 (2010).

[3] V. Alexandrov, M. Dzero, and P. Coleman, Phys. Rev. Lett. 111, 226403 (2013).

[4] M. Sundermann, H. Yavaş, K. Chen, D. J. Kim, Z. Fisk, D. Kasinathan, M. W. Haverkort, P. Thalmeier, A. Severing, and L. H. Tjeng, Phys. Rev. Lett. 120, 016402 (2018).

[5] D.-J. Kim, J. Xia, and Z. Fisk, Nat. Mater. 13, 466 (2014).

[6] D.-J. Kim, S. Thomas, T. Grant, J. Botimer, Z. Fisk, and J. Xia, Sci. Rep. 3, 3150 (2013).

[7] M. Neupane, N. Alidoust, S.-Y. Xu, T. Kondo, Y. Ishida, D. J. Kim, C. Liu, I. Belopolski, Y. J. Jo, T.-R. Chang et al., Nat. Commun. 4, 2991 (2013).

[8] J. Jiang et al., Nat. Commun. 4, 3010 (2013).

[9] N. Xu et al., Nat. Commun. 5, 4566 (2014).

[10] S. Wolgast, Ç. Kurdak, K. Sun, J. W. Allen, D.-J. Kim, and Z. Fisk, Phys. Rev. B 88, 180405(R) (2013).

[11] P. Hlawenka et al., Nat. Commun. 9, 517 (2018).

[12] B. S. Tan et al., Science 349, 287 (2015).

[13] G. Li et al., Science 346, 1208 (2014).

[14] S. M. Thomas, X. Ding, F. Ronning, V. Zapf, J. D. Thompson, Z. Fisk, J. Xia, and P. F. S. Rosa, Phys. Rev. Lett. 122, 166401 (2019).

[15] Y. S. Eo, A. Rakoski, J. Lucien, D. Mihaliov, C. Kurdak, P. F. S. Rosa, and Z. Fisk, Proc. Natl. Acad. Sci. USA 116, 12638 (2019).

[16] B. Gorshunov, N. Sluchanko, A. Volkov, M. Dressel, G. Knebel, A. Loidl, and S. Kunii, Phys. Rev. B 59, 1808 (1999).

[17] N. J. Laurita, C. M. Morris, S. M. Koohpayeh, P. F. S. Rosa, W. A. Phelan, Z. Fisk, T. M. McQueen, and N. P. Armitage, Phys. Rev. B 94, 165154 (2016).

[18] N. J. Laurita et al., Physica B (Amsterdam, Neth.) 536, 78 (2019).

[19] M. E. Valentine, S. Koohpayeh, W. A. Phelan, T. M. McQueen, P. F. S. Rosa, Z. Fisk, and N. Drichko, Phys. Rev. B 94, 075102 (2016).

[20] M. E. Valentine, S. Koohpayeh, W. A. Phelan, T. M. Queen, P. F. S. Rosa, Z. Fisk, and N. Drichko, Physica B (Amsterdam, Neth.) 536, 60 (2018).

[21] M. H. Hamidian et al., Proc. Natl. Acad. Sci. USA 108, 18233 (2011).

[22] R. Sollie and P. Schlottmann, J. Appl. Phys. 69, 5478 (1991).

[23] P. Schlottmann and C. S. Hellberg, J. Appl. Phys. 79, 6414 (1996).
[24] J. Figgins and D. K. Morr, Phys. Rev. Lett. 107, 066401 (2011),

[25] H.-F. Lü, H.-Z. Lu, S.-Q. Shen, and T.-K. Ng, Phys. Rev. B 87, $195122(2013)$

[26] A. Arab, A. X. Gray, S. Nemsak, D. V. Evtushinsky, C. M. Schneider, D.-J. Kim, Z. Fisk, P. F. S. Rosa, T. Durakiewicz, and P. S. Riseborough, Phys. Rev. B 94, 235125 (2016).

[27] H. Pirie et al., Nat. Phys. 16, 52 (2020).

[28] L. Jiao, S. Rößler, D. Kasinathan, P. F. S. Rosa, C. Guo, H. Yuan, C.-X. Liu, Z. Fisk, F. Steglich, and S. Wirth, Sci. Adv. 4, eaau4886 (2018).

[29] W. T. Fuhrman, J. R. Chamorro, P. A. Alekseev, J.-M. Mignot, T. Keller, J. A. Rodriguez-Rivera, Y. Qiu, P. Nikolić, T. M. McQueen, and C. L. Broholm, Nat. Commun. 9, 1539 (2018).

[30] W. T. Fuhrman and P. Nikolić, Phys. Rev. B 101, 245118 (2020).

[31] G. Wiese, H. Schäffer, and B. Elschner, Europhys. Lett. 11, 791 (1990).

[32] J. C. Souza, P. F. S. Rosa, U. Burkhardt, M. König, Z. Fisk, P. G. Pagliuso, S. Wirth, and J. Sichelschmidt, JPS Conf. Proc. 30, 011021 (2020).

[33] S. Kunii, T. Uemura, Y. Chiba, T. Kasuya, and M. Date, J. Magn. Magn. Mater. 52, 271 (1985).

[34] K. Kojima, M. Kasaya, and Y. Koi, J. Phys. Soc. Jpn. 44, 1124 (1978).

[35] G. Feher and A. F. Kip, Phys. Rev. 98, 337 (1955).

[36] F. J. Dyson, Phys. Rev. 98, 349 (1955).

[37] S. Barnes, Adv. Phys. 30, 801 (1981).

[38] Y. S. Eo, S. Wolgast, A. Rakoski, D. Mihaliov, B. Y. Kang, M. S. Song, B. K. Cho, M. Ciomaga Hatnean, G. Balakrishnan, Z. Fisk, S. R. Saha, X. Wang, J. Paglione, and Ç. Kurdak, Phys. Rev. B 101, 155109 (2020).

[39] S. Sen, N. S. Vidhyadhiraja, E. Miranda, V. Dobrosavljević, and W. Ku, Phys. Rev. Research 2, 033370 (2020).

[40] M. Orendáč, S. Gabáni, G. Pristáš, E. Gažo, P. Diko, P. Farkašovský, A. Levchenko, N. Shitsevalova, and K. Flachbart, Phys. Rev. B 96, 115101 (2017).

[41] J. M. Lawrence, T. Graf, M. F. Hundley, D. Mandrus, J. D. Thompson, A. Lacerda, M. S. Torikachvili, J. L. Sarrao, and Z. Fisk, Phys. Rev. B 53, 12559 (1996).

[42] G. G. Lesseux, P. F. S. Rosa, Z. Fisk, P. Schlottmann, P. G. Pagliuso, R. R. Urbano, and C. Rettori, AIP Adv. 7, 055709 (2017).

[43] See Supplemental Material at http://link.aps.org/supplemental/ 10.1103/PhysRevResearch.2.043181 for additional magnetic susceptibility data and $\mathrm{Eu}^{2+}$ ESR spectra.

[44] A. Abragam and B. Bleaney, Electron Paramagnetic Resonance of Transition Ions (Clarendon, Oxford, 1970). 
[45] T. Uemura, Y. Chiba, M. Hagiwara, and M. Date, J. Phys. Soc. Jpn. 55, 3737 (1986).

[46] T. S. Altshuler and M. S. Bresler, J. Exp. Theor. Phys. 88, 1019 (1999).

[47] K. Baberschke and E. Tsang, Phys. Rev. Lett. 45, 1512 (1980).

[48] P. Schlottmann, Magnetochemistry 4, 27 (2018).

[49] B. I. Kochelaev, Low Temp. Phys. 43, 76 (2017).

[50] H. A. Buckmaster and Y. H. Shing, Phys. Status Solidi A 12, 325 (1972).

[51] H. Sturm, B. Elschner, and K.-H. Höck, Phys. Rev. Lett. 54, 1291 (1985).

[52] P. W. Anderson and P. R. Weiss, Rev. Mod. Phys. 25, 269 (1953).

[53] F. A. Garcia, P. A. Venegas, P. G. Pagliuso, C. Rettori, Z. Fisk, P. Schlottmann, and S. B. Oseroff, Phys. Rev. B 84, 125116 (2011).

[54] P. A. Venegas, F. A. Garcia, D. J. Garcia, G. G. Cabrera, M. A. Avila, and C. Rettori, Phys. Rev. B 94, 235143 (2016).

[55] R. R. Urbano, C. Rettori, G. E. Barberis, M. Torelli, A. Bianchi, Z. Fisk, P. G. Pagliuso, A. Malinowski, M. F. Hundley, J. L. Sarrao, and S. B. Oseroff, Phys. Rev. B 65, 180407(R) (2002).

[56] G. A. Wigger, C. Beeli, E. Felder, H. R. Ott, A. D. Bianchi, and Z. Fisk, Phys. Rev. Lett. 93, 147203 (2004).

[57] R. R. Urbano, P. G. Pagliuso, C. Rettori, P. Schlottmann, J. L. Sarrao, A. Bianchi, S. Nakatsuji, Z. Fisk, E. Velazquez, and S. B. Oseroff, Phys. Rev. B 71, 184422 (2005).

[58] A. Rakoski, Y. S. Eo, K. Sun, and Ć. Kurdak, Phys. Rev. B 95, 195133 (2017).

[59] B. Skinner, Phys. Rev. Mater. 3, 104601 (2019).

[60] S. Yeo, K. Song, N. Hur, Z. Fisk, and P. Schlottmann, Phys. Rev. B 85, 115125 (2012).

[61] G. G. Lesseux et al., J. Phys.: Condens. Matter 28, 125601 (2016).
[62] L. L. Hirst, Phys. Kondens. Mater. 11, 255 (1970).

[63] T. Gambke, B. Elschner, and L. L. Hirst, Phys. Rev. Lett. 40, 1290 (1978).

[64] P. A. Venegas and G. E. Barberis, Phys. Rev. B 46, 911 (1992).

[65] C. Rettori, S. B. Oseroff, D. Rao, P. G. Pagliuso, G. E. Barberis, J. Sarrao, Z. Fisk, and M. Hundley, Phys. Rev. B 55, 1016 (1997).

[66] J. G. S. Duque, E. M. Bittar, C. Adriano, C. Giles, L. M. Holanda, R. Lora-Serrano, P. G. Pagliuso, C. Rettori, C. A. Pérez, R. Hu, C. Petrovic, S. Maquilon, Z. Fisk, D. L. Huber, and S. B. Oseroff, Phys. Rev. B 79, 035122 (2009).

[67] P. G. Pagliuso, C. Rettori, J. L. Sarrao, A. Cornelius, M. F. Hundley, Z. Fisk, and S. B. Oseroff, Phys. Rev. B 60, 13515 (1999).

[68] D. Davidov, C. Rettori, A. Dixon, K. Baberschke, E. P. Chock, and R. Orbach, Phys. Rev. B 8, 3563 (1973).

[69] W. T. Fuhrman, J. C. Leiner, J. W. Freeland, M. van Veenendaal, S. M. Koohpayeh, W. A. Phelan, T. M. McQueen, and C. Broholm, Phys. Rev. B 99, 020401(R) (2019).

[70] S. Kunii, K. Takeuchi, I. Oguro, K. Sugiyama, A. Ohya, M. Yamada, Y. Koyoshi, M. Date, and T. Kasuya, J. Magn. Magn. Mater. 52, 275 (1985).

[71] H. Shen and L. Fu, Phys. Rev. Lett. 121, 026403 (2018).

[72] R. Zhang, B. Singh, C. Lane, J. Kidd, Y. Zhang, B. Barbiellini, R. S. Markiewicz, A. Bansil, and J. Sun et al., arXiv:2003.11052 (2020).

[73] A. Barla, J. Derr, J. P. Sanchez, B. Salce, G. Lapertot, B. P. Doyle, R. Rüffer, R. Lengsdorf, M. M. Abd-Elmeguid, and J. Flouquet, Phys. Rev. Lett. 94, 166401 (2005).

[74] Y. Zhou, P. F. S. Rosa, J. Guo, S. Cai, R. Yu, S. Jiang, K. Yang, A. Li, Q. Si, Q. Wu, Z. Fisk, and L. Sun, Phys. Rev. B 101, $125116(2020)$. 